\title{
Prednisolone Induced Cushing Syndrome: A Case Report
}

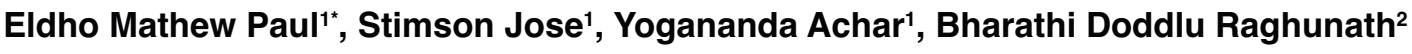 \\ 1'Department of Pharmacy Practice, SJMCP, Chitradurga-577501, Karnataka, INDIA. \\ 2Department of Pharmacology, SJMCP, Chitradurga-577501, Karnataka, INDIA.
}

\begin{abstract}
Chronic use of steroids have a lot of serious side effects like Cushing syndrome. Here is a case of 39 year old female patients who is suffering from Cushing syndrome caused by chronic use of prednisolone. She is a known case of rheumatoid arthritis since six months and she is taking prednisolone. She has complaints of moon face, backache, swelling of limbs, abdominal distension, muscle weakness and striaes since 3 weeks. Her serum cortisol level is elevated. She was treated with treated with furosemide, oral hypoglycemic agents, anti-ulcer drugs. The prednisolone dose is tapered.
\end{abstract}

Key words: Prednisolone, Cushing syndrome, Rheumatoid arthritis, Cortisol, Moon face, Steroids.

\section{INTRODUCTION}

Cushing syndrome is a condition characterized by high blood levels of cortisol or other exogenous compounds of glucocorticoids. This can be iatrogenic or endogenous cortisol secretion, due to the either an adrenal tumor or hyper secretion of adrenocorticotropic hormone by the pituitary gland. The pathophysiological mechanism differs based on the cause of Cushing syndrome. Prolonged administration of exogenous glucocorticoid hormones especially in the treatment of lymph proliferative disorders at levels of higher doses are the most common cause of Cushing syndrome. The most common systemic side effects of prolonged use of glucocorticoids includes Cushing's syndrome, cataract, hypertension, dyslipidemia, skin atrophy, failure to thrive, hypo-thalamopituitary-adrenal axis suppression, striae, glaucoma and a predisposition to life-threatening infections. ${ }^{1}$

\section{CASE HISTORY}

A 39 Year old female patient was admitted in general medicine department of Basveshwara Medical Collage Hospital and Research
Center, Chitradurga with the chief complaints of moon face, backache, swelling of limbs, abdominal distension, muscle weakness and striaes since 3 weeks. She is a known case of rheumatoid arthritis since six month and on regular treatment with prednisolone $30 \mathrm{mg}$ daily. The patient was conscious and well oriented to time and place but on physical appearance was looking weak, pale and his vitals were as follows BP-160/10 mm of Hg, PR-77 bpm, CVS-S1,S2+ ,RS -NVBS $+\mathrm{VE}$, CNS- no abnormality present, P/Adistension+

The laboratory investigations shows that the patients had elevated fasting blood glucose level $(180 \mathrm{mg} / \mathrm{dl})$, serum cortisol level was elevated to $33.4 \mathrm{mcg} / \mathrm{dl}$, serum potassium level is reduced to $3.3 \mathrm{mmol} / \mathrm{L}$. serum triglyceride levels are elevated to $207 \mathrm{mg} / \mathrm{dl}$. So based on subjective and objective evidence, the patient is provisionally diagnosed as having Cushing syndrome due to the chronic use of prednisolone. The patient was treated with furosemide, oral hypoglycemic agents, anti-ulcer drugs. The prednisolone dose is tapered and the serum cortisol level is measured after 3 months during follow up
DOI: 10.5530/ijopp.9.2.15




visit which shows reduction in the serum cortisol level. Based on this evidence, the patient is finally diagnosed as exogenous Cushing's syndrome due to chronic use of prednisolone.

\section{DISCUSSION}

Cushing syndrome may be due to the endogenous causes such as ectopic Adrenocorticotropic hormone (ACTH) production, pituitary tumor, and exogenous causes like exogenously administration of corticosteroids. Exogenous Cushing's syndromeis a condition resulting from long-term exposure to therapeutic administration of corticosteroids. They are also known as steroid- induced Cushing's syndrome or iatrogenic Cushing syndrome. ${ }^{1}$ Patients with Cushing's disease usually present with one or more signs and symptoms secondary to the presence of excess cortisol or ACTH. ${ }^{2}$ Unless the patient is taking a corticosteroid, serum cortisol levels are low in exogenous Cushing's syndrome.

Most patients with Cushing syndrome have round, red, full moon face, growth retardation in children, fat accumulation on the trunk along with weight gain, fat loss from the buttocks, legs, and arms (central obesity), skin infections, purple marks (striae) on the skin of the breast, abdomen and thighs , thinly skin with easy bruising, backache while doing routine activities, fat deposit between the shoulders and above the collar bone, hips and shoulder muscle weakness, fracture of the rib and spine due to thinning of the bones.

The common laboratory findings in Cushing syndrome includes lack of response to an ACTH stimulation test, low ACTH level, elevated fasting blood sugar level, decreased serum potassium level, lower bone density, increased blood cholesterol levels etc.

Treatment is done by tapering the dose of corticosteroids which may take an year. Sudden stoppage corticosteroids after chronic intake can results in adrenal crisis. Slowly tapering the corticosteroid that is causing Cushing syndrome can help reverse the effects of adrenal gland atrophy. $^{3}$

Glucocorticoids are commonly used in clinical practice for the management of various autoimmune, inflammatory and allergic diseases. Irrational administration of glucocorticoids are most common particularly in chronic therapies which leads to many side effects like hypothalamic-pituitary-adrenal axis suppression, Cushing's syndrome, increased risk of infections and changes in mental status. The pharmacokinetic properties of the glucocorticoids, individual difference in steroid metabolism, daily dosage and the duration of treatment are the factors influencing therapeutic and adverse effect of glucocorticoids. ${ }^{4}$

Before initiating steroid therapy, patients should be well informed about the possible side effects of steroids. Otherwise it may leads to severe systemic side effects including Cushing's syndrome, hypertension, dyslipidemia, suppression of hypothalamic-pituitary-adrenal axis, striae, glaucoma, skin atrophy, cataract and predisposition to life-threatening infections. ${ }^{5}$

\section{CONCLUSION}

Chronic use of synthetic corticosteroids such as prednisolone is the most common cause of Cushing syndrome. Patient/caretaker should be thoroughly informed about the potential risk associated with its long term use and the symptoms of steroid induced illness. The patient should undergo regular medical checkups to assess the effect of steroid in the body.

\section{ACKNOWLEDGEMENTS}

We take this opportunity to express our gratitude and respectful thanks to all the faculty members who give support and assistance to publish this case report.

\section{CONFLICT OF INTEREST}

The author declares there is no conflict of interest.

\section{ABBREVIATION USED}

ACTH: Adrenocorticotropic Hormone; BP: Blood Pressure; CNS: Central Nervous System; CVS: Cardio Vascular System; NVBS: Non Vesicular Breath Sound.

\section{REFERENCES}

1. Siddarama R, Reddy $\mathrm{YH}$, Reddy GA. A case report on steroid induced Cushing syndrome and NSAID induced bronchial asthma. IAJPR. 2015;5(4):1404-07.

2. Kirk LF, Hash RB. Cushing's Disease: Clinical Manifestations and Diagnostic Evaluation. Am Fam Physician. 20001;62(5):1119-27.

3. Wisse $B$, Zieve D, Black B. Cushing syndrome - exogenous. US national library of medicine. American Accreditation HealthCare Commission.

4. Romanholi DJ, Salqadol LR. Arq Bras Endocrinol Metabol. 2007;51(8):1280-92.

5. West DP, Micali G. Principles of pediatric dermatological therapy. In: Harper J, Oranje A, Prose N, editors. Textbook of Pediatric Dermatology. $1^{\text {st }}$ ed. London: Blackwell Science Ltd. 2000;1731-42. 